\title{
Accuracy analysis of the modular fixturing systems with grid pattern threaded holes
}

\author{
Tudor Păunescu ${ }^{1, *}$, and Rodica Păunescu ${ }^{1}$ \\ ${ }^{1}$ Transilvania University of Brasov, Department of Manufacturing Engineering, Mihai Viteazu No. 5, \\ Braşov, Romania.
}

\begin{abstract}
Using a baseplate with a grid pattern threaded holes and the machine tool to accurately locate the modular locators upon the baseplate, the positional accuracy of the workpiece can be improved and results a relatively inexpensive modular fixturing systems. A stochastic mathematical model of the geometrical accuracy and a solving algorithm for these modular fixturing systems are proposed. Four types of modular fixturing systems with grid pattern threaded versus the most important classical modular-fixturing systems with multipurpose holes are analysed. Monte Carlo approach is used to calculate and to compare the locating accuracy of these modular fixturing systems.
\end{abstract}

\section{Introduction}

Two primary forms of modular fixturing systems (MFS) are available: with grid pattern holes and with T-slots. The first system offers several advantages over second: more positional possibilities, more security, repeated setups are easier, if grid pattern holes are damaged, these are repairable [1]. Commercial grid-pattern modular fixturing systems are based on alternating dowel and tapped holes or multipurpose holes (MFS-MH). The last system has an alignment bushing and threaded insert in each hole, so a multipurpose hole can be used as an alignment hole, mounting hole, or can have both functions.

The present paper focuses on the MFS with a particularity: baseplates have a grid pattern with threaded holes (MFS-TH) and the machine tool is used to accurately locate the modular components upon the baseplate.

The idea to use machine tool to setup modular work holdings is not a new one. Over the past decades several boring machines were equipped with a tool (fig. 1a) to accurately align the locating modules (fig. 1b).

In fig. 1c is shown an example of prismatic part located with 3-2-1 method: the machine tool plate is the primary surface, the two modules locate part on secondary surface and one locating module restricts the last degree of freedom. The screws and clamps are not shown.

\footnotetext{
* Corresponding author: t.paun@unitbv.ro
} 

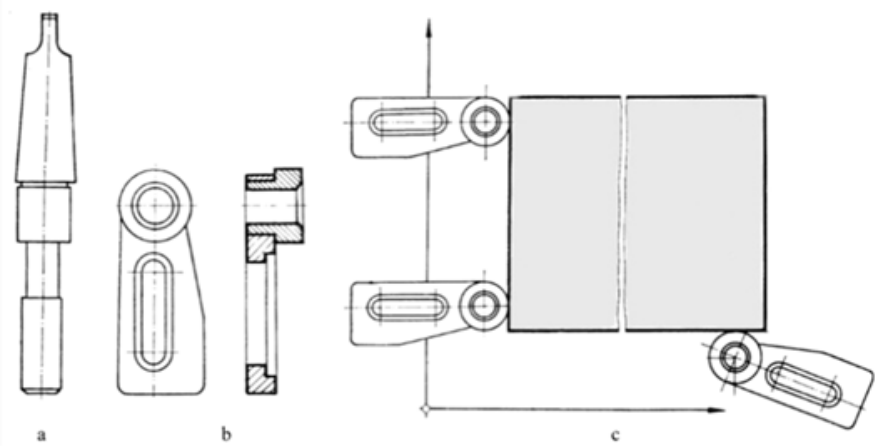

Fig. 1. The tool (a) used to accurately align the locating module (b), an example of 3-2-1 locating scheme (c) [2].

Other modular fixturing system using machine tool to setup locators is proposed by $\mathrm{P}$.

V. Hudimac [3].

The author claims that [3]:

- MFS-TH eliminates the need to accurately align the grid-pattern reference frame of the baseplate with the coordinate system reference frame of the machine tool.

- MFS-TH eliminates the dependence upon the manufactured precision of the baseplate, resulting an inexpensive baseplate.

- The stacking-up of modular fixturing components are free of errors accumulation.

- Locating and clamping elements can be easily assembled and disassembled.
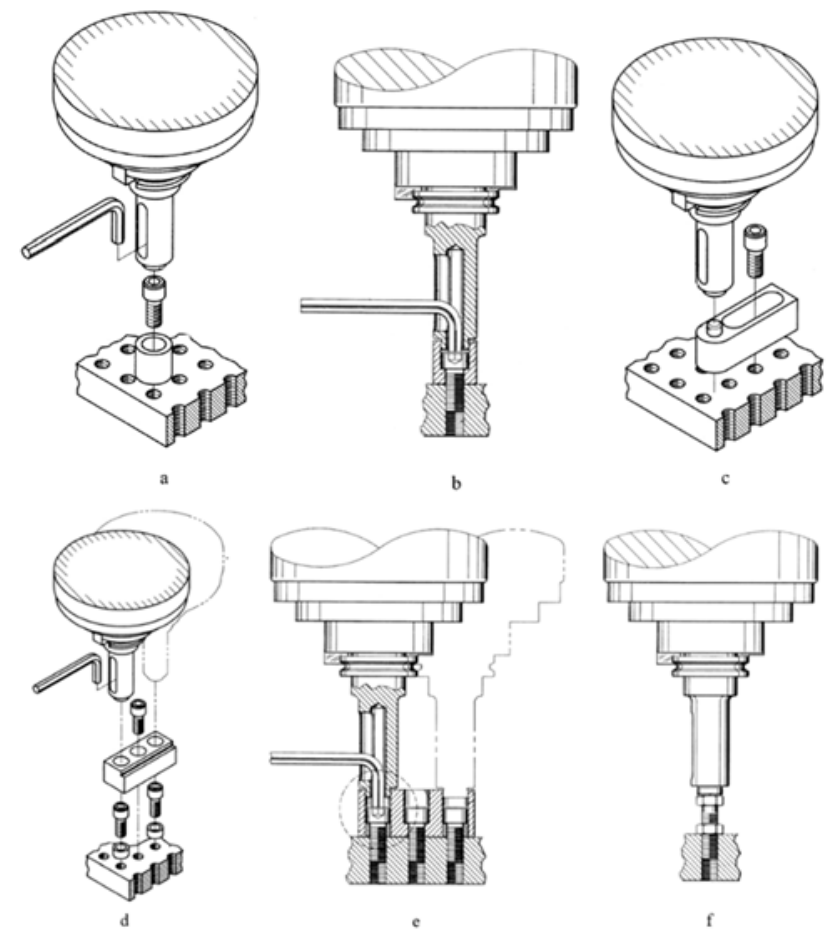

Fig. 2. How is assembled a cylindrical locating module (a-c), a single edge locator (d, e) and an adjustable support (f) [3]. 
As you see in fig. 2a-e, the locating tool is positioned within a machine tool spindle, the nose is accurately centered in the counterbore of the locating module and wrench is engaged in the head of screw. Wrench is turned to engage fastener into the threaded hole of the baseplate and, finally the locating module is affixed into position. In fig. $2 \mathrm{f}$ the end surface of tool contacts the top surface of the threaded adjustable support to set the Z-axis workpiece reference surface. In our opinion, the cylindrical locating module (fig. 2a, b, c) can be easily and accurately assembled, but is more difficult to accurately assemble the locating modules with two holes (fig. $2 \mathrm{~d}$ and e).

Both solutions described above use cylindrical pin and hole with clearance fit, to accurately align locators. The clearance between the locating tool nose and module counter bore is a source of positional error. If the centering surfaces are conical the clearance is zero and this source of error is eliminated. For example, two types of locators with conical surfaces, and two modular fixtures are shown in fig. 3 [4].
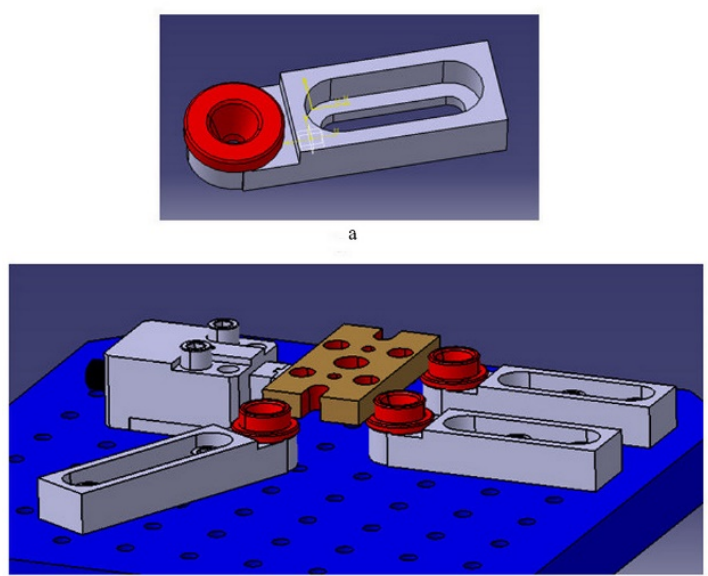

c

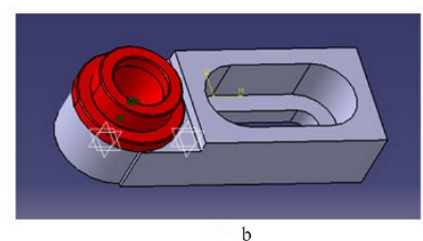

b

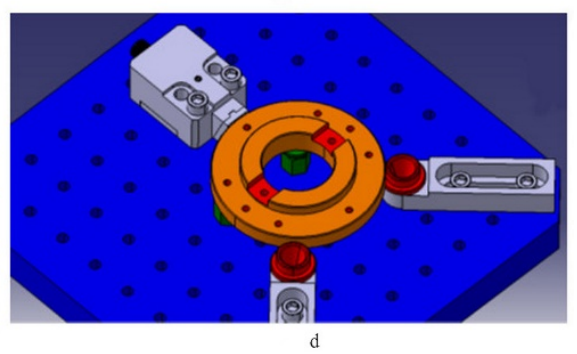

Fig. 3. Two types of modular locators (a, b) and examples of modular fixtures (c, d) [4].
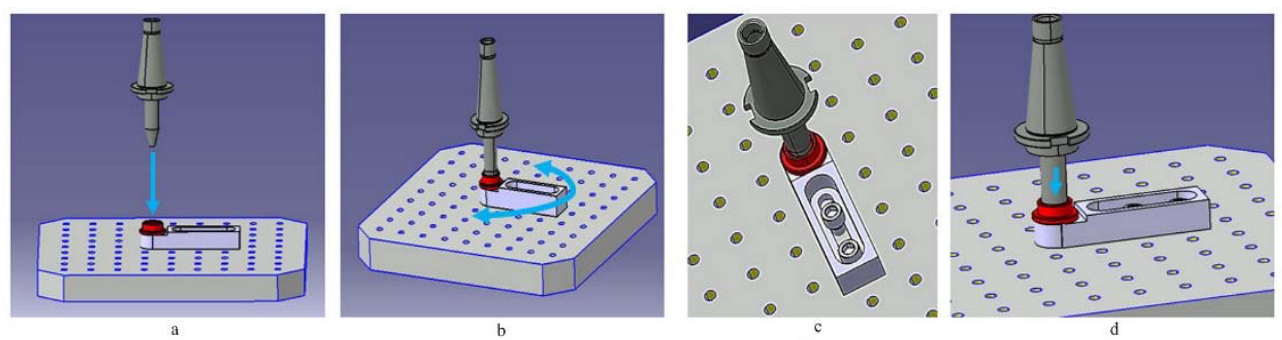

Fig. 4. Steps of locator-baseplate assembly [4].

Steps of locator-baseplate assembly, shown in fig.4, are described below:

- The modular locator is placed in an approximate position on the baseplate, the tool is lowered to programmed target. A clearance between the tool and sleeve is necessary for rotational adjustment of the locator.

- The locator is manually rotated to fit to threaded holes.

- Screws are tighten with low forces.

- The tool is lowered in the final position until the clearance between the tool and sleeve is zero, then the screws are tighten again. 


\section{Sources of geometrical errors and modelling}

If the machine tool is used to accurately locate the modular components upon the baseplate, a number of error sources contribute to the resulting workpiece location error, which can lead to poor part quality:

- machine tool positioning inaccuracy;

- inaccuracy of the tool holder;

- inaccuracy of the locating tool;

- clearance between locating tool nose and the locator hole.

One of the most important error of the machine tool is the quasi-static geometric/kinematic error. The geometric errors may be compensated, a lot of machine tool manufacturers provide the errors mapping and a subsequent compensation in the same manner as has been well established in CMMs [5]. Now, most modern controllers have the capability of volumetric positioning error compensation.

For a 3 -axis machine, there are 6 errors per axis, a total of 21 errors $(6 \times 3$ plus 3 squareness errors): linear displacement errors, vertical and horizontal straightness errors, roll, pitch and yaw angular errors and squareness errors.

Machine tool accuracy has generally been described by the linear positioning accuracy and repeatability of the axes, but this is a deficient specification because other geometric effects: angular, straightness and squareness errors are ignored. A more comprehensive way to define precision would be to specify the accuracy for the full working volume of a machine tool taking into account all geometric errors [6].

Traditionally, manufacturers have ensured accuracy of parts with linear calibration of each axis of the machine tools, but this method is inadequate for ensuring accuracy of 3D parts. The conventional definition of the $3 \mathrm{D}$ volumetric positioning error is the root mean square of the 3 axes displacement error. This approach is correct if the dominate errors are the displacement errors.

In our days the displacement errors are reduced considerably, the dominate errors are straightness and squareness errors. ASME B5.54 and ISO 230-6 standards are working on a new definition of volumetric accuracy. A complete measurement of those 21 errors is very complex and time consuming, for those reasons the body diagonal displacement error is a good quick check of the volumetric error [7, 8].

Machine tool builders list accuracy and repeatability, but often is not given the method used. A number of standards exist but differ in their analysis procedures and in key parameter definition (ISO 230-1 2012, ASME B5.54, JIS B 6336-1986). As a result the values reported for positional accuracy and repeatability can vary depending on which standard was used $[10,11]$. More, if the same workpiece is machined at different locations of the work table, different positioning errors will be obtained [12].

In our approach the following hypotheses are considered:

- errors are quasi-static and geometric;

- vertical machine centers are used to align the locators;

- because the movement on $\mathrm{Z}$ axis is short the straightness and squareness errors are neglected, so a 2D model is proposed, and the spatial errors are projected on XY plane;

- geometric error of the cylindrical locator axis is related to machine coordinate system (machine zero).

Errors are summed as vectors (fig. 5b), chained tip-to-tail. In statistical tolerance design each vector-error has two parameters: magnitude and angle [9]. The first parameter describes the distance of the axis relative to its true position, and the second dictates the direction. It is assumed that the random variable angle is a uniform distribution from 0 to $2 \pi$. 
In fig. 5a is shown the worst aligning case of the locator (variant 1 in table 1): the locating tool is mounted in the tool holder (2), a clearance exists between the tool (3) and locator (4).

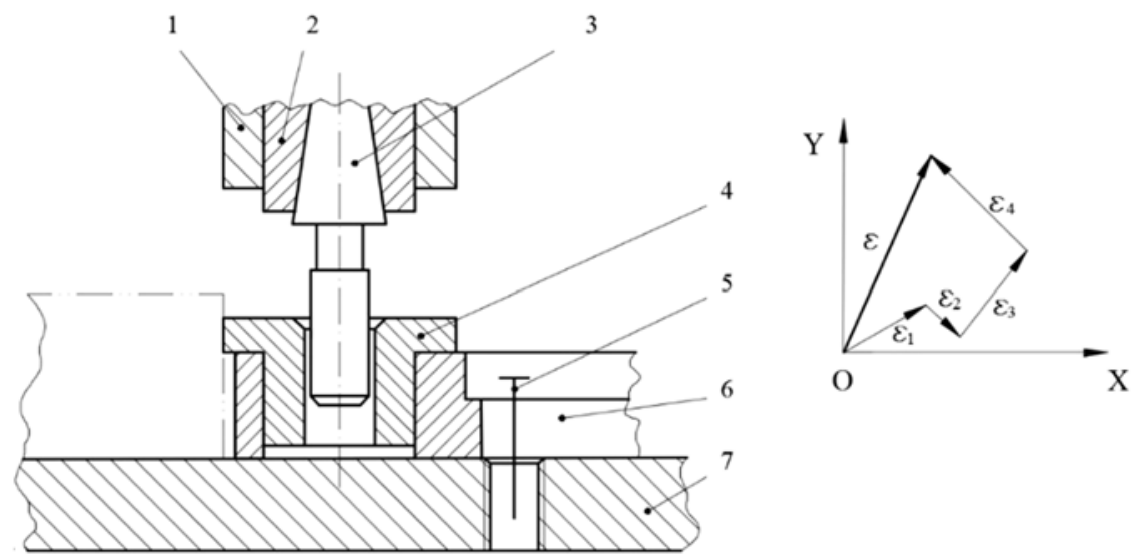

Fig. 5. Drawing section on subassembly locator-aligning tool (a), summed errors as vectors (b).

Because the centering surface can be cylindrical or conical, and the tool holder is used or not, four variants of locating schemes can be generated (table 1).

Table 1. Aligning schemas of the cylindrical modular locators.

\begin{tabular}{|c|l|c|c|c|c|}
\hline \multirow{2}{*}{ No } & \multicolumn{2}{|c|}{ Source of error } & \multicolumn{4}{c|}{ Variant } \\
\cline { 3 - 6 } & & $\mathbf{1}$ & $\mathbf{2}$ & $\mathbf{3}$ & $\mathbf{4}$ \\
\hline $\mathbf{1}$ & Machine tool positioning inaccuracy $\left(\boldsymbol{\varepsilon}_{1}\right)$ & $\mathrm{x}$ & $\mathrm{x}$ & $\mathrm{x}$ & $\mathrm{x}$ \\
\hline $\mathbf{2}$ & Inaccuracy of the tool holder $\left(\boldsymbol{\varepsilon}_{2}\right)$ & $\mathrm{x}$ & $\mathrm{x}$ & - & - \\
\hline $\mathbf{3}$ & Inaccuracy of the locating tool $\left(\boldsymbol{\varepsilon}_{3}\right)$ & $\mathrm{x}$ & $\mathrm{x}$ & $\mathrm{x}$ & $\mathrm{x}$ \\
\hline $\mathbf{4}$ & $\begin{array}{l}\text { Clearance between locating tool nose and } \\
\text { the locator hole }\left(\mathbf{\varepsilon}_{\mathbf{4}}\right)\end{array}$ & $\mathrm{x}$ & - & $\mathrm{x}$ & - \\
\hline
\end{tabular}

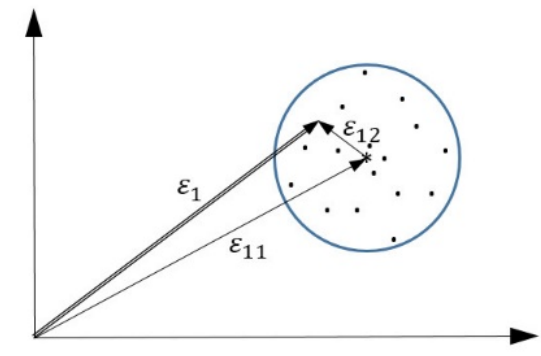

Fig. 6. Machine tool positioning accuracy $\left(\boldsymbol{\varepsilon}_{11}\right)$ and repeatability $(\boldsymbol{\varepsilon} 12)$ summed as vectors. 
Machine tool positioning accuracy ( $\left.\boldsymbol{\varepsilon}_{1}\right)$ has two components (fig.6): positioning accuracy $\left(\boldsymbol{\varepsilon}_{11}\right)$ and repeatability $\left(\boldsymbol{\varepsilon}_{12}\right)$. Today, the positioning accuracy of medium size vertical machine centers is $\pm 0.002 \ldots \pm 0.005 \mathrm{~mm}$ and generally the repeatability is half of the accuracy: $\pm 0.001 \ldots \pm 0.003 \mathrm{~mm}$.

The second source is inaccuracy of the tool holder $\left(\boldsymbol{\varepsilon}_{2}\right)$. The runout of tool holders is under $\mathrm{ro}_{2}=0.01 \mathrm{~mm}$, but runout of the new generation of high-accuracy tool holders is 0.003 $\mathrm{mm}$.

Inaccuracy of the locating tool $\left(\varepsilon_{3}\right)$ is the third source of positioning error, the runout is $\mathrm{ro}_{3}=0.005 \ldots 0.01 \mathrm{~mm}$.

If the centering surface of the locating tool is cylindrical, fits H6/g5 and H7/g6 having a minimum clearance are reasonable.

\section{Monte Carlo simulation}

Generally, the geometrical accuracy analysis of the MFS is a difficult task because modular locating schemes and the dimensional chains are spatial, multi-dimensional, nonlinear and often in presence of clearances.

Compared with other methods Monte Carol simulation (MCS) offers many advantages: simplicity, flexibility, it can be used for nonlinear assembly functions, other distributions than the normal may describe the fixture modules dimension better and more easily. But MCS analysis has two disadvantages: the computational effort can be large, so hundreds of thousands of samples or more are required for accurate results and MCS cannot allocate optimal tolerances.

The problem: estimating the statistical positioning accuracy of the modular locator positioned by means of machine-tool spindle.

In case of variant 1 (see table 1 ) the variables are:

- machine tool positioning inaccuracy $\left(\boldsymbol{\varepsilon}_{1}\right)$ : positioning accuracy $\left(\boldsymbol{\varepsilon}_{11}\right)$ and repeatability $\left(\boldsymbol{\varepsilon}_{12}\right)$ are $2 \mathrm{D}$ vectors, first has a constant magnitude and the second is a random variable with a normal or uniform distribution from 0 to repeatability, both have directions uniform distributed from 0 to $2 \pi$;

- inaccuracy of the tool holder $\left(\boldsymbol{\varepsilon}_{2}\right)$ and inaccuracy of the locating tool $\left(\boldsymbol{\varepsilon}_{3}\right)$ are vectors with magnitudes $0-\mathrm{ro}_{2} / 2$, respectively $0-\mathrm{ro}_{3} / 2$, and angles have a uniform distribution from 0 to $2 \pi$;

- $\boldsymbol{\varepsilon}_{4}$ is generated by the clearance between the tool (4) and locator (3) $c=D_{4}-d_{3}$; usually the diameters of the centering surfaces have normal distributions or uniform, magnitude is random variable with a uniform distribution from 0 to $\mathrm{c} / 2$ and the direction is random variables with a uniform distribution from 0 to $2 \pi$.

The proposed algorithm simulates the $2 \mathrm{D}$ positioning process:

- The error-vector $\boldsymbol{\varepsilon}_{1}$ is generated as result of adding $\boldsymbol{\varepsilon}_{11}$ and $\boldsymbol{\varepsilon}_{12}$.

- The error-vectors $\varepsilon_{2}$ and $\varepsilon_{3}$ are calculated.

- The last error-vector $\boldsymbol{\varepsilon}_{4}$ is generated.

- $\boldsymbol{\varepsilon}_{\mathbf{i}}, \mathrm{i}=1-4$ are chained heads to tails.

- The steps $1 \ldots 4$ are repeated, sample size is $n=10^{6}$.

- Resulting errors are statistical processed, the positioning error of the locator is calculated based on $\pm 3 \sigma$.

For variant $2 \varepsilon_{4}=0$, for $3 \varepsilon_{2}=0$ and for $4 \varepsilon_{2}=0$ and $\varepsilon_{4}=0$.

Results of the Monte Carlo simulations using Mathcad are shown in table 2. Three cases are considered: high, medium and low positioning accuracies. For each case, in the first row of the table 2 random variables with uniform distributions are considered, in the second row accuracy and repeatability of machine tool have uniform distributions and other ones have normal distributions. 
Table 2. Results of MFS-TH Monte Carlo simulations.

\begin{tabular}{|c|c|c|c|c|c|}
\hline \multirow{2}{*}{ No } & \multirow{2}{*}{ Specifications } & \multicolumn{4}{|c|}{$\begin{array}{l}\text { Positioning accuracy of the modular locator } \\
\qquad \varepsilon_{x}=\varepsilon_{y}, \varepsilon_{r}[\mu \mathrm{m}]\end{array}$} \\
\hline & & Var.1 & Var.2 & Var.3 & Var.4 \\
\hline \multirow{2}{*}{1} & \multirow{2}{*}{$\begin{array}{l}\text { High accuracy: } \\
\text { acc }=2 \mu \mathrm{m}, \mathrm{rep}=1 \mu \mathrm{m}, \\
\text { ro }_{2}=\mathrm{ro}_{3}=3 \mu \mathrm{m}, \mathrm{H} 6 / \mathrm{g} 5\end{array}$} & $\pm 12,13.7$ & $\pm 6.3,7.1$ & $\pm 11.4,12.9$ & $\pm 4.9,5.4$ \\
\hline & & $\pm 10.4,11.7$ & $\pm 4.9,5.5$ & $\pm 10.1,11.3$ & $\pm 4.1,4.7$ \\
\hline \multirow{2}{*}{2} & \multirow{2}{*}{$\begin{array}{l}\text { Medium accuracy: } \\
\mathrm{acc}=3 \mu \mathrm{m}, \text { rep }=1.5 \mu \mathrm{m} \\
\mathrm{ro}_{2}=\mathrm{ro}_{3}=5 \mu \mathrm{m}, \mathrm{H} 6 / \mathrm{g} 5\end{array}$} & $\pm 14.5,16.6$ & $\pm 10.2,11.5$ & $\pm 13.2,15$ & $\pm 7.8,8.5$ \\
\hline & & $\pm 12.2,13.8$ & $\pm 7.7,8.5$ & $\pm 11.6,13$ & $\pm 6.6,7.5$ \\
\hline \multirow{2}{*}{3} & \multirow{2}{*}{$\begin{array}{l}\text { Low accuracy: } \\
\text { acc }=5 \mu \mathrm{m}, \mathrm{rep}=2.5 \mu \mathrm{m}, \\
\mathrm{ro}_{2}=\mathrm{ro}_{3}=10 \mu \mathrm{m}, \mathrm{H} 7 / \mathrm{g} 6\end{array}$} & $\pm 24.1,27.4$ & $\pm 19.5,21.9$ & $\pm 20.8,23.7$ & $\pm 14.4,15.7$ \\
\hline & & $\pm 19.2,21.9$ & $\pm 14.5,16.6$ & $\pm 17.6,20$ & $\pm 12.1,13.9$ \\
\hline
\end{tabular}

In every cell of the table 2 the first value is positioning accuracy on $\mathrm{X}$ and $\mathrm{Y}$ axis $\left(\varepsilon_{x}=\right.$ $\varepsilon_{y}$, fig.7), the second value is the half of positional error $\left(\varepsilon_{r i}=\sqrt{\varepsilon_{x i}^{2}+\varepsilon_{y i}^{2}}, i=1-n\right)$.

If $10^{6}$ sample size is used, the stability of results is very good: for second variant, medium precision and uniform distribution the positioning error varies $\pm 0.4 \%$.

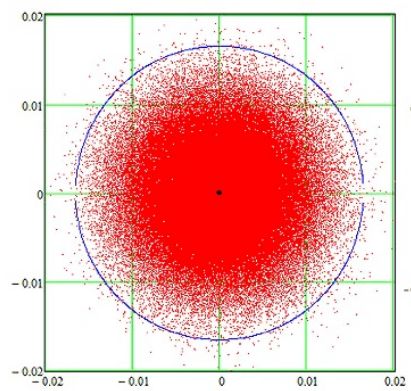

1

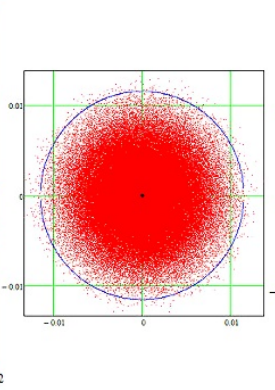

2

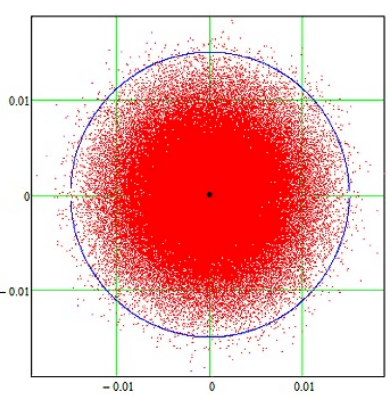

3

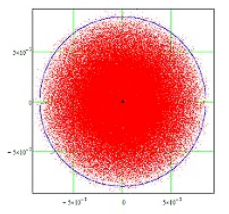

4

Fig. 7. Locator positioning accuracy for medium precision, uniform distributions (row 3, table 2).

First of all, influence of clearance between locating tool nose and the locator hole is greater than accuracy of machine tool, roundness of the centering tool and tool holder (variants 1, 2 and 3, 4, table 2).

If the centering surfaces are conical the clearance is zero, but the locator aligning is more difficult.

Secondly, machine tool positioning accuracy and repeatability are a significant sources of errors. 


\section{Comparison between MFS-TH and MFS-MH}

MFS-MHs (threaded and cylindrical locating surfaces are coaxial) use cylindrical locating surface of screws/sleeves to accurately locate the modular locators. In contrast to MFS-TH the locating modules of MFS-MH are aligned outside of machine tool.

The mathematical model and solving of the MFS-MH are similar with MFS-TH, but the datum is the baseplate frame.

Table 3. Results of Monte Carlo simulations for couple of commercial MFS-MHs.

\begin{tabular}{|c|c|c|c|c|c|}
\hline MFS-MH & Pin/Sleeve & Hole 1 & Hole 2 & Pitch & $\varepsilon_{x}=\varepsilon_{y}, \varepsilon_{r}[\mu m]$ \\
\hline \multirow{2}{*}{ Halder L12 } & \multirow{2}{*}{$12 \mathrm{k} 5$} & \multirow{2}{*}{$12 \mathrm{~F} 6$} & \multirow{2}{*}{12 F6 } & \multirow{2}{*}{$50 \pm 0.01$} & $\pm 18.6,21.3$ \\
\hline & & & & & $\pm 17.0,19.2$ \\
\hline \multirow{2}{*}{ KippM12 } & \multirow{2}{*}{$12 \mathrm{~g} 5$} & \multirow{2}{*}{$12 \mathrm{~F} 7$} & \multirow{2}{*}{$12 \mathrm{~F} 7$} & \multirow{2}{*}{$50 \pm 0.01$} & $\pm 25.3,28.8$ \\
\hline & & & & & $\pm 23.5,26.3$ \\
\hline \multirow[t]{2}{*}{ Nabeya M12 } & \multirow{2}{*}{$12_{0.01}^{0.021}$} & \multirow{2}{*}{$12 \begin{array}{l}0.009 \\
0.004\end{array}$} & \multirow{2}{*}{$12 \begin{array}{l}0.009 \\
0.004\end{array}$} & \multirow{2}{*}{$80 \pm 0,01$} & $\pm 10.8,12.5$ \\
\hline & & & & & $\pm 9.0,10.3$ \\
\hline \multirow[t]{2}{*}{ AMF M12 } & \multirow{2}{*}{$12 \begin{array}{l}0.011 \\
0\end{array}$} & \multirow{2}{*}{12 F7 } & \multirow{2}{*}{$12 \mathrm{~F} 7$} & \multirow{2}{*}{$40 \pm 0.01$} & $\pm 19.9,23.1$ \\
\hline & & & & & $\pm 17.6,20.0$ \\
\hline
\end{tabular}

Sources of errors are: position tolerance of the grid pattern, clearances between the centering hole of fixture base and screw/sleeve, and between mounted locator and screw/sleeve.

The input variables are: position tolerance of grid pattern (TPp), upper and lower specification limits of holes and screw/sleeve, respectively. Two different distributions of input data were tested: normal and uniform. The sample size is $10^{6}$ to get a reliable measure of the output distribution. Data analysis of Monte Carlo simulations from table 2 and 3 shows that fixtures based on MFS-TH can be more accurate than MFS-MH.

\section{Conclusion}

MFS-THs are feasible in the job and batch productions. Because MFS-THs eliminate the need to align the grid-pattern of the baseplate with coordinate reference frame of the machine tool and the dependence upon the manufactured precision of the baseplate, fixtures assembled online are cheaper than fixtures assembled offline from modules with multipurpose holes.

Most locators of the MFS-THs are cylindrical locators, these can be quickly and accurately aligned with a centering tool mounted in the machine tool shaft. Aligning the more complex locators than cylindrical locators is time expensive and inaccurate, moreover modular blocks can't be mounted, so with these modules only a limited number of workholdings can be assembled.

In this paper several MFS-THs are analysed, and a new variant with conical centering surface is proposed.

A general stochastic model of the MFS-TH locating accuracy is proposed, and it is solved using Monte Carlo simulation.

The positional accuracy of four MFS-THs with low, medium and high precision are calculated. Monte Carlo simulations show that with components of MFS-THs can be assembled accurate modular fixtures.

Even MFS-THs currently aren't used, these MFSs can be more accurate and cheaper than MFS-MHs. 


\section{References}

1. ***, Modular Fixturing Handbook (Carr Lane Mfg. Co. St. Louis, Missouri, 1991)

2. E. Botez, Tehnologii de programare numerică a mașinilor unelte [Numerical Programming Technologies of the Machine Tools] (Technical Publishing House, Bucharest, 1973)

3. P.V. Hudimac, Machine-set modular-fixturing system. Patent US5509214 (1996)

4. B. Brânzoi, Dispozitive de prindere modulare setabile pe centre de prelucrare [Modular Fixtures Configured by Machining Centers] (M.S. Thesis, Transilvania University of Brasov, 2015)

5. J. Gu, 43rd Proceedings of the North American Manufacturing Research $\mathbf{x x x},(2015)$

6. S. Fletcher, Laser Metrology and Machine Performance (Euspen Ltd, Cranfield University, 2009)

7. O. Svoboda, P. Bach, IMTS 2004 Manufacturing Conference, 1 (2004)

8. C. Wang, Seven International Symposium on Instrumentation and Control Technology, ISICT08, 7129, (2008)

9. S. H. Huang, Int. J. Prod. Res. 42, 4871 (2004)

10. S. Klabunde, R.Schmidt, Modern Machine Shop 3, (1998)

11. B. Mullany, Transactions of NAMRI/SME, 36, 309 (2008)

12. K. Gupta, 5th International \& 26th All India Manufacturing Technology, Design and Research Conference (AIMTDR), 1 (2014) 